\title{
Interleukine und Wachstumsregulation von
} Melanomzellen

\author{
Interleukins and Regulation of Growth of Melanoma Cells
}

\section{Zusammenfassung}

Im Rahmen eigener experimentellen Untersuchungen in vitro gelang es nachzuweisen, dass IL-8 von humanen Melanomzellen sezerniert wird. Die Blockade der IL-8-Freisetzung mittels neutralisierender Antikörper oder alternativ molekularbiologisch durch Zugabe von IL-8-Sequenz-spezifischen Antisense-Oligonukleotiden belegten die Bedeutung von IL-8 als potenter Wachstumsfaktor für Melanomzellen, der sowohl parakrin als auch autokrin wirken kann. In-situ-Hybridiserungen an Primärtumoren und benignen melanozytären Kontrollen wiesen auf eine gewisse prognostische Relevanz des IL-8-Nachweises beim Melanom hin. Im Nacktmausmodell war durch andere Gruppen eine klare Assoziation mit Wachstumsdynamik und Metastasierung nachweisbar, die in neusten Untersuchungen mit einem humanisierten, IL-8-neutralisierenden Antikörper therapeutisch günstigt beeinflusst werden konnte. Es bleibt abzuwarten, ob dieser Erfolg versprechende Antikörper in naher Zukunft erste klinische Studien erreicht.

\section{Abstract}

In a series of in-vitro experiments our group demonstrated that human melanoma cells secrete soluble IL- 8 . Blockade of IL-8 secretion either by neutralizing antibodies or by molecular biological techniques such as antisense oligomers led to a pronounced growth inhibition of melanoma cells and confirm the role of IL-8 as a paracrine and/or autocrine growth factor. Further in-situ hybridisation on primary melanomas as well as benign melanocytic nevi revealed a correlation between IL-8 expression and cinical parameters such as time-to-progression. Additional work by other groups confirmed a strong association between IL-8 and tumor growth and metastasis in a nude mouse model. In the same model a fully humanized IL-8 neutralizing antibody was able to significantly alter the clinical outcome. It remains to be seen whether these promising antibodies will find their way into the clinic in the near future.

\section{Einleitung}

Das Melanom ist ein maligner Tumor neuroektodermalen Ursprungs mit steigender Inzidenz und Mortalität. Bekanntermaßen ist die frühzeitige Erkennung und Exzision der einzige Garant für eine gute Prognose, da im fortgeschrittenen Krankheits- stadium die Heilungschancen aufgrund der hohen Therapieresistenz minimal sind [1]. Andererseits werden beim Melanom oft immunologisch vermittelte Phänomene beobachtet. Die Überlegung, dass ein verbessertes pathoätiologisches Verständnis möglicherweise alternative Therapiekonzepte beflügeln könnte, bildete die Basis, Anfang der neunziger Jahre die Rolle von Zytoki- 


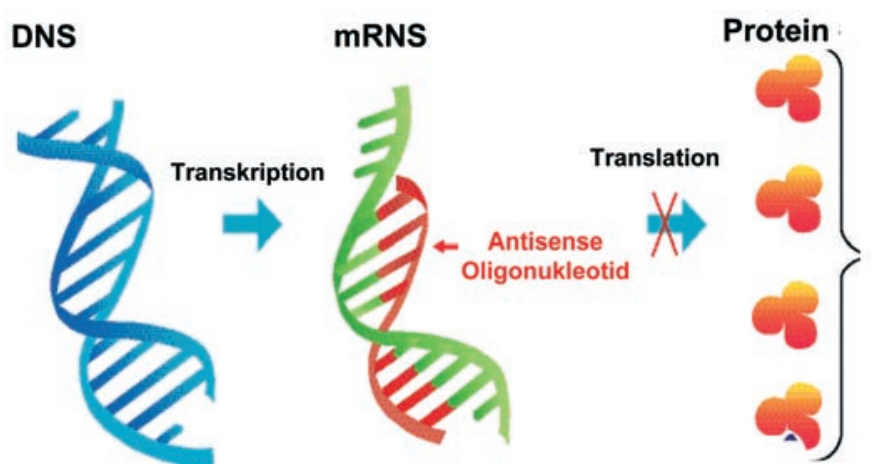

Abb. 1 Prinzip der Inhibition der Proteinsynthese durch Zugabe von Antisense-Oligonukleotidsträngen, von ca. 20 Aminosäuren Länge, die sich sequenzspezifisch an die mRNS anlagern und hochselektiv mit der Translation eines definierten Proteins interferieren.

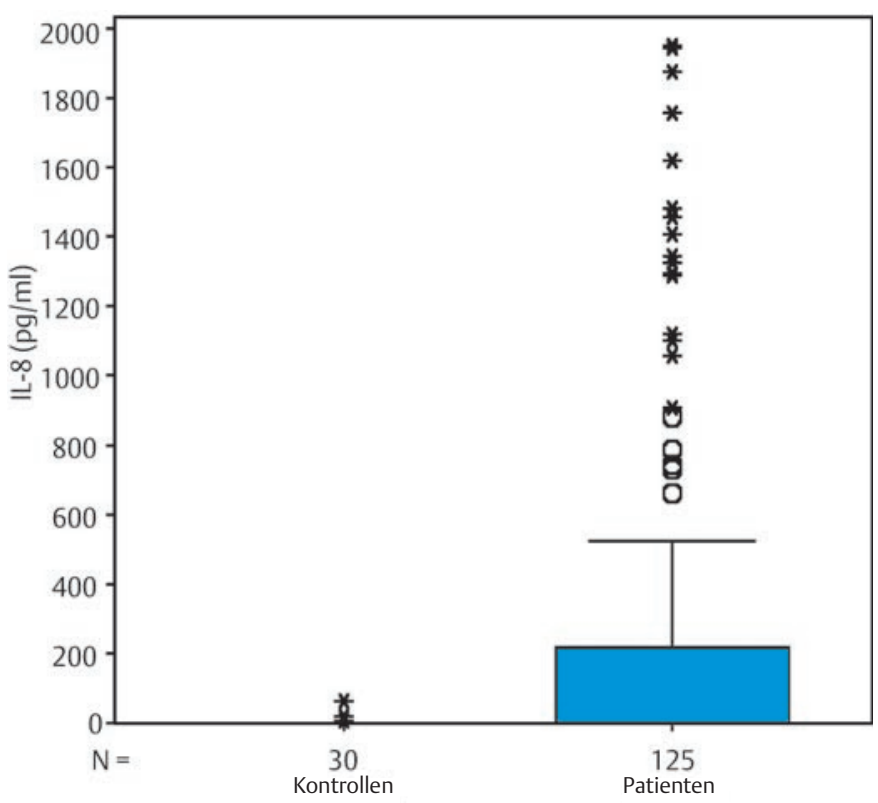

Abb. 2 Nachweis von Interleukin-8 aus Seren von 30 Kontrollpersonen und 125 Melanompatienten in unterschiedlichen Tumorstadien. Der beobachtete Unterschied ist hoch statistisch signifikant; $P=0,004$ (modifiziert nach Ugurel et al., 2001).

nen als proliferativ und antiproliferativ wirkende Faktoren auf das Melanomzellwachstum zu untersuchen.

Im Rahmen der Arbeiten für den ersten Förderpreis der Berliner Dermatologischen Gesellschaft galt unser Interesse insbesondere Interleukin-8 (IL-8) [2]. IL-8 war Ende der achtziger Jahre von der Arbeitsgruppe um Prof. J. Schröder, Kiel, als ein von aktivierten Monozyten freigesetzter Mediator mit erheblicher Potenz auf Neutrophile beschrieben worden [3]. Darüber hinaus war relativ schnell klar, dass IL-8 ein breit proinflammatorisch wirksames Zytokin ist, was von verschiedenen Zelltypen freigesetzt werden kann. Ziel der preisgekrönten Arbeiten war es herauszufinden, inwiefern IL-8 als Wachstumsfaktor für Melanomzellen Bedeutung hat.
Als erster Schritt wurde untersucht, ob Melanomzellen IL-8 selbst freisetzen. Dazu wurden mittels ELISA-Technologie Zellkultur-Überstände von 8 humanen Melanomlinien in vitro analysiert. Dabei zeigte sich, dass 6/8 der Melanomlinien (jeweils 1 Mio. Zellen) IL- 8 zwischen 0,4 bis $14,9 \mathrm{ng} / \mathrm{ml}$ freisetzen. Nachfolgend zeigte sich erwartungsgemäß, dass die IL-8-Sekretion durch Aktivierung der Zellen mit fötalem Serum, PMA oder IL- $1 \alpha+\beta$ weiter zu steigern war (bis zu $500 \%$ ). Auf Ebene der RNS-Expression konnte IL-8 als auch die beiden IL-8-Rezeptoren (heutige Nomenklatur: CXCR1 und CXCR2) mittels PolymeraseKettenreaktion (PCR) nachgewiesen werden.

Um die Kausalität der IL-8 vermittelten biologischen Phänomene präziser untersuchen zu können, haben wir dann so genannte Oligonukleotide synthetisieren lassen, die komplementär zur IL-8 RNS waren (sog. Antisense-Oligonukleotide), die sich Sequenz-spezifisch anlagern und die Translation des spezifischen Proteins behindern und stark reduzieren. Das Prinzip der Antisense-Strategie ist in Abb.1 dargestellt. Im Vergleich zu einer Reihe von Kontrollsequenzen zeigte sich dann in den verschiedenen Versuchen, dass IL-8-Antisense-Oligonukeotide, die ins Medium gegeben wurden, dosisabhängig aufgenommen wurden und die Proteinsynthese des Interleukin-8 beeinflußten. So gelang es, die Sekretion von IL-8 in den Kulturüberstand weitestgehend zu reduzieren, als auch das klonale Wachstum in einem Weichagarsystem durch IL-8-Antisense-Oligonukleotide zu beeinflussen. Dies konnte in einer unabhängigen Versuchsserie auch mit blockierenden Antikörpern bestätigt werden. Zusammenfassend gelang es uns mit den 1993 veröffentlichen Daten erstmals zu zeigen, dass IL-8 ein wichtiger essenzieller, autokriner Wachstumsfaktor für humane Melanomzellen darstellte [2].

\section{Weitere Entwicklung und klinischer Praxisbezug}

Die Tatsache, dass IL-8 relativ einfach in die Umgebung als löslicher Faktor freigesetzt werden kann, machte diesen Mediator auch interessant, ob dieser als „Tumormarker“ von Bedeutung sein könnte. Bereits früh wurde IL-8 im Serum von Melanompatienten als erhöht und mit der Tumorlast assoziiert beschrieben [4]. Dies konnte durch Ugurel et al. [11] in einer umfassenden Studie bezüglich der klinischen und prognostischen Bedeutung verschiedener angiogenetischer Faktoren im Serum von 125 Melanompatienten bestätigt werden (Abb.2). Allerdings ist die Messung von IL-8 im Serum nicht Melanom-spezifisch, da eine Vielzahl anderer Zellen und zusätzlich im Rahmen inflammatorischer Prozesse IL-8 freigesetzt wird, was IL-8 für eine verlässliche Diagnostik oder ein Therapiemonitoring nur schwer einsetzbar macht. So hat sich die Bestimmung von IL-8 im Serum bei Melanompatienten bislang auch nicht durchgesetzt.

Eigene weitergehende Untersuchungen hatten das Ziel zu erurieren, inwieweit IL-8-Expression in den Tumoren in situ möglicherweise von prognostischer Relevanz wäre. Dazu untersuchten wir 37 Primärmelanome und 22 melanozytäre Nävi mittels In-situ-Hybridisierung [5]. Dabei zeigte sich, dass IL-8-Expression - entweder im Primärtumor selbst oder in der periläsionalen Haut - mit einer verkürzten Zeit bis zum Auftreten neuer Meta- 
stasen assoziiert war. Diese Ergebnisse standen in guter Übereinstimmung mit Daten aus einem Nacktmausmodell von Singh und Mitarbeitern. Singh et al. [6] belegten, dass die Proteinsekretion von IL-8 in 13 verschiedenen humanen Melanomlinien gut mit ihrer Fähigkeit in Nacktmäusen zu wachsen und zu metastasieren korreliert war. Die Zugabe von rekombinant hergestelltem IL-8 stimulierte in der Versuchsserie die Proliferation der wenigmetastasierenden A375P-Zelllinie. Die selbe Gruppe konnte weiterhin zeigen, dass die IL-8-Rezeptorexpression (CXCR1 und CXCR2) ebenfalls von kritischer Bedeutung für das Wachstum im Nacktmausmodell ist [7].

Heute weiß man, dass IL-8 durch viele Zelltypen auch in der unmittelbaren Tumorumgebung, z.B. Endothelzellen, produziert wird, und dass so neben der direkten IL-8/Melanomzell-Interaktion auch eine große Zahl indirekter und parakriner Effekte eine Rolle spielt, deren Zusammenfassung hier den Rahmen sprengen würde. Interessanterweise konnte gezeigt werden, dass IL-8 wie auch andere angiogene Faktoren nach Zellkontakt mit Zytostatika hochreguliert wird und so möglicherweise den Zytostatikabedingten therapeutischen Nutzen konterkariert $[8,9]$.

Dennoch - oder gerade deshalb - ist IL-8 heute weiterhin eine Zielstruktur, die auch für therapeutische Ansätze versucht wird zu nutzen. So ist es der Arbeitsgruppe um Bar-Eli gelungen, einen vollständig humanisierten IL-8-neutralisierenden Antikörper (ABX-IL-8) herzustellen, der im präklinischen Tiermodell sehr Erfolg versprechend erscheint. Wieder in einem Nacktmausmodell gelang es durch Injektion von ABX-IL-8 das Melanomtumorwachstum erheblich zu unterdrücken und die Metastasierungsfrequenz zu reduzieren. Diese Phänomene waren durch eine reduzierte Aktivität von Metalloproteasen (MMP-2) und verminderte Neoangiogenese sowie gesteigerte Apoptose bedingt [10].

\section{Fazit}

IL-8 hat sich als potenter Wachstumsfaktor für Melanomzellen erwiesen, der sowohl parakrin als auch autokrin wirken kann. Im Nacktmausmodell ist eine klare Assoziation mit Wachstumsdynamik und Metastasierung von humanen Melanomen nachweisbar, die in neuesten Untersuchungen mit einem humanisierten, IL-8-neutralisierendem Antikörper therapeutisch günstig beeinflusst werden kann. Es bleibt abzuwarten, ob dieser Antikörper in naher Zukunft erste klinische Studien erreicht.
Danksagung

Mein Dank für die jahrelange gute und fruchtbare Kooperation, ohne die diese Ergebnisse nicht möglich gewesen wären, gilt der DFG sowie den Dres. W. Nürnberg, M. Worm, B. Algermissen und B.-M. Czarnetzki/Henz.

\section{Literatur}

${ }^{1}$ Schadendorf D. Is there a standard for the palliative treatment of melanoma? Onkologie 2002; 25: $74-76$

2 Schadendorf D, Möller A, Algermissen B, Worm M, Sticherling M, Czarnetzki BM. IL-8 produced by human malignant melanoma cells in vitro is an essential autocrine growth factor. J Immunol 1993; 151: 2667-2675

${ }^{3}$ Schröder JM, Mrowietz U, Morita E, Christophers E. Purification and partial biochemical characterization of a human moncyte-derived neutrophil-activating peptide that lacks interleukin-1 activity. J Immunol 1987; 139: $3474-3483$

${ }^{4}$ Scheibenbogen C, Mohler T, Haefele J, Hunstein W, Keilholz U. Serum interleukin-8 (IL-8) is elevated in patients with metastatic melanoma and correlates with tumour load. Melanoma Res 1995; 5: 179-181

${ }^{5}$ Nürnberg W, Tobias D, Otto F, Henz BM, Schadendorf D. Expression of interleukin-8 detected by in situ hybridization correlates with worse prognosis in primary cutaneous melanoma. J Pathol 1999; 189: $546-$ 551

${ }^{6}$ Singh RK, Gutman M, Radinsky R, Bucana CD, Fidler IJ. Expression of interleukin 8 correlates with the metastatic potential of human melanoma cells in nude mice. Cancer Res 1994; 54: 3242 - 3247

${ }^{7}$ Varney ML, Li A, Dave BJ, Bucana CD, Johansson SL, Singh RK. Expression of CXCR1 and CXCR2 receptors in malignant melanoma with different metastatic potential and their role in interleukin-8 (CXCL-8)mediated modulation of metastatic phenotype. Clin Exp Metastasis 2003; 20: $723-731$

${ }^{8}$ Lev DC, Ruiz M, Mills L, McGary EC, Price JE, Bar-Eli M. Dacarbazine causes transcriptional up-regulation of interleukin 8 and vascular endothelial growth factor in melanoma cells: a possible escape mechanism from chemotherapy. Mol Cancer Ther 2003; 2: 753 - 763

${ }^{9}$ Lev DC, Onn A, Melinkova VO, Miller C, Stone V, Ruiz M, McGary EC, Ananthaswamy HN, Price JE, Bar-Eli M. Exposure of melanoma cells to dacarbazine results in enhanced tumor growth and metastasis in vivo. J Clin Oncol 2004; 22: 2092 - 2100

${ }^{10}$ Huang S, Mills L, Mian B, Tellez C, McCarty M, Yang XD, Gudas JM, BarEli M. Fully humanized neutralizing antibodies to interleukin-8 (ABXIL8) inhibit angiogenesis, tumor growth, and metatstasis of human melanoma. Am J Pathol 2002; 161: 125-134

${ }^{11}$ Ugurel S, Rappl G, Tilgen W, Reinhold U. Increased serum concentration of angiogenic factors in malignant melanoma patients correlates with tumor progression and survival. J Clin Oncol 2001; 19: 577-583 